\title{
The number of transmission channels through a single-molecule junction
}

\author{
J. P. Bergfield \\ College of Optical Sciences, University of Arizona, 1630 East University Boulevard, AZ 8572 用 \\ J. D. Barr and C. A. Stafford \\ Department of Physics, University of Arizona, 1118 East Fourth Street, Tucson, AZ 85721
}

\begin{abstract}
We calculate transmission eigenvalue distributions for Pt-benzene-Pt and Pt-butadiene-Pt junctions using realistic state-of-the-art many-body techniques. An effective field theory of interacting $\pi$-electrons is used to include screening and van der Waals interactions with the metal electrodes. We find that the number of dominant transmission channels in a molecular junction is equal to the degeneracy of the molecular orbital closest to the metal Fermi level.
\end{abstract}

The transmission eigenvalues $\tau_{n}$ constitute a mesoscopic PIN code [1] characterizing quantum transport through any nanoscale device. For a single-atom contact between two metallic electrodes, the number of transmission channels is simply given by the chemical valence of the atom [2]. Recently, highly-conductive single-molecule junctions (SMJ) with multiple transport channels have been formed from benzene molecules between Pt electrodes [3]. This raises the question if there exists a similarly simple criterion determining the number of transmission eigenchannels in a SMJ.

Previous calculations [4, 5] using effective singleparticle models based on density functional theory appear to answer the above question in the negative. However, we find that transmission channel distributions calculated using many-body theory do yield a simple, intuitive answer to this important question.

For a two-terminal SMJ, $\tau_{n}$ are eigenvalues of the elastic transmission matrix [4-7]

$$
T(E)=\Gamma_{1}(E) G(E) \Gamma_{2}(E) G^{\dagger}(E),
$$

where $G$ is the retarded Green's function [8] of the SMJ and $\Gamma_{\alpha}$ is the tunneling-width matrix describing the coupling of the molecule to lead $\alpha$. The number of transmission channels is equal to the rank of the matrix (1), which is in turn limited by the ranks of the matrices $\Gamma_{\alpha}$ and $G$. The additional two-fold spin degeneracy of each resonance is considered implicit. The rank of $\Gamma_{\alpha}$ is equal to the number of covalent bonds formed between the molecule and lead $\alpha$. Thus, for example, in a SMJ where a Au electrode bonds to an organic molecule via a thiol group, only a single bond is formed, and there is only one non-negligible transmission channel [5, 9]. In Pt-benzene-Pt junctions, however, each $\mathrm{Pt}$ electrode forms multiple bonds to the benzene molecule [3].

In this article, we investigate how transmission eigenvalue distributions of SMJs depend on the number of lead-molecule bonds and on molecular symmetry using a many-body theory of transport [8]. Specifically, we focus on junctions with benzene $\left(\mathrm{C}_{6} \mathrm{H}_{6}\right)$ and butadiene $\left(\mathrm{C}_{4} \mathrm{H}_{6}\right)$ bonded to two Pt leads (see Fig. 11). Consistent with refs. 3 and 5, we find that the total number of nonzero transmission eigenvalues in a SMJ is limited only by the number of

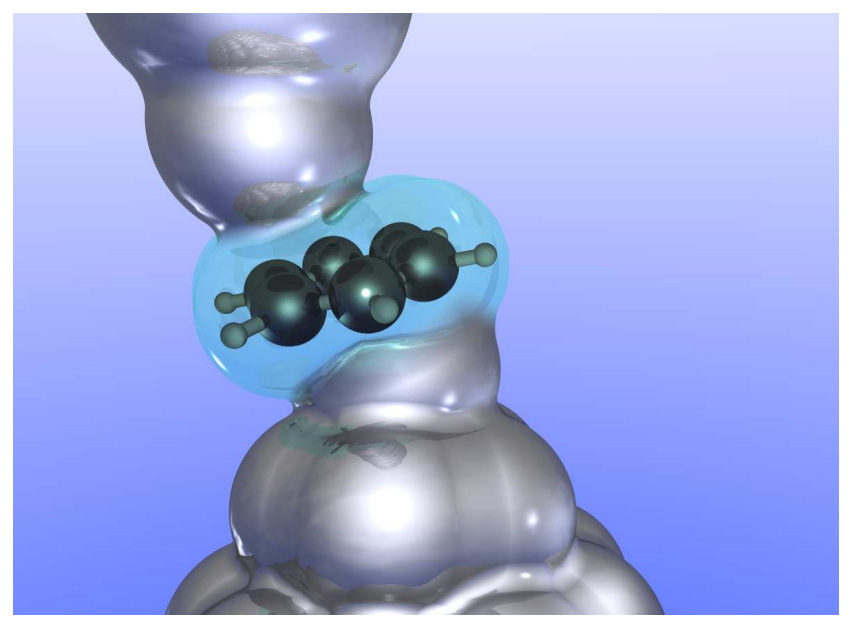

FIG. 1. Schematic diagram of a Pt-benzene-Pt junction. The lead-molecule coupling is mediated predominantly by individual $\mathrm{Pt}$ atoms at the tips of each electrode.

bonds to each electrode. However, increasing the number of bonds past a certain point leads to additional channels with very small transmission $\left\langle\tau_{n}\right\rangle \ll 1$. The central finding of this article is that in SMJs with sufficient numbers of lead-molecule bonds the number of dominant transmission channels is equal to the degeneracy of the molecular orbital closest to the metal Fermi level. Additional transmission channels stemming from further off-resonant molecular states are strongly suppressed, but may still be experimentally resolvable [3] for very strong lead-molecule hybridization.

\section{MANY-BODY THEORY OF TRANSPORT}

When macroscopic leads are attached to a single molecule, a SMJ is formed, transforming the few-body molecular problem into a true many-body problem. Until recently, a theory of transport in SMJs that properly accounts for both the particle and wave character of the electron has been lacking, so that the Coulomb blockade and coherent transport regimes were considered 
'complementary.' [10] In this article, we utilize a nonequilibrium many-body theory [8] that correctly accounts for wave-particle duality, reproducing the key features of both the Coulomb blockade and coherent transport regimes. Previous applications [8, 11-13] to SMJs utilized a semiempirical Hamiltonian [14] for the $\pi$-electrons, which accurately describes the gas-phase spectra of conjugated organic molecules. This approach should be adequate to describe molecules weakly coupled to metal electrodes, e.g. via thiol linkages. However, in junctions where the $\pi$ electrons bind directly to the metal electrodes [3], the leadmolecule coupling may be so strong that the molecule itself is significantly altered, necessitating a more fundamental molecular model. To address this issue, we have developed an effective field theory of interacting $\pi$-electrons, in which the form of the molecular Hamiltonian is derived from symmetry principles and electromagnetic theory (multipole expansion), rather than using an ad-hoc parametrization [14]. The resulting formalism constitutes a state-ofthe-art many-body theory that provides a realistic description of lead-molecule hybridization and van der Waals coupling, as well as the screening of intramolecular interactions by the metal electrodes, all of which are essential for a quantitative description of strongly-coupled SMJs [3].

The Green's function of a SMJ has the form [8]

$$
G(E)=\left[G_{\text {mol }}^{-1}(E)-\Sigma_{\mathrm{T}}(E)-\Delta \Sigma_{\mathrm{C}}(E)\right]^{-1},
$$

where $G_{\mathrm{mol}}$ is the molecular Green's function, $\Sigma_{\mathrm{T}}$ is the tunneling self-energy matrix, whose imaginary part is given by $\operatorname{Im} \Sigma_{\mathrm{T}}=-\sum_{\alpha} \Gamma_{\alpha} / 2$, and $\Delta \Sigma_{\mathrm{C}}$ is the correction to the Coulomb self-energy due to the broadening of the molecular resonances in the junction. At room temperature and for small bias voltages, $\Delta \Sigma_{\mathrm{C}} \approx 0$ in the cotunneling regime [8] (i.e., for nonresonant transport). Furthermore, the inelastic transmission probability is negligible compared to Eq. 1 in that limit.

The molecular Green's function $G_{\mathrm{mol}}$ is found by exactly diagonalizing the molecular Hamiltonian, projected onto a basis of relevant atomic orbitals [8]:

$$
G_{\mathrm{mol}}(E)=\sum_{\nu, \nu^{\prime}} \frac{\left[\mathcal{P}(\nu)+\mathcal{P}\left(\nu^{\prime}\right)\right] C\left(\nu, \nu^{\prime}\right)}{E-E_{\nu^{\prime}}+E_{\nu}+i 0^{+}},
$$

where $E_{\nu}$ is the eigenvalue associated with eigenstate $\nu$ of the molecular Hamiltonian, $\mathcal{P}(\nu)$ is the probability that the state $\nu$ is occupied, and $C\left(\nu, \nu^{\prime}\right)$ is a rank-1 matrix with elements

$$
\left[C\left(\nu, \nu^{\prime}\right)\right]_{n \sigma, m \sigma^{\prime}}=\left\langle\nu\left|d_{n \sigma}\right| \nu^{\prime}\right\rangle\left\langle\nu^{\prime}\left|d_{m \sigma^{\prime}}^{\dagger}\right| \nu\right\rangle .
$$

Here $d_{n \sigma}$ annihilates an electron of spin $\sigma$ on the $n$th atomic orbital of the molecule. For linear response, $\mathcal{P}(\nu)$ is given by the grand canonical ensemble. Equations 24 imply that each molecular resonance $\nu \rightarrow \nu^{\prime}$ contributes at most one transmission channel in Eq. 1 .

An effective field theory of interacting $\pi$-electrons was used to model the electronic degrees of freedom most relevant for transport. Using symmetry principles and an electrostatic multipole expansion, an effective Hamiltonian for

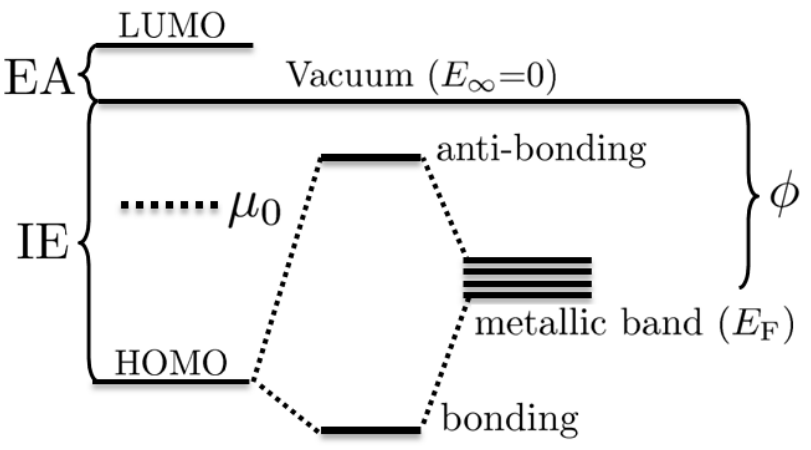

FIG. 2. Energy-level diagram of a molecule with ionization energy IE and electron affinity EA bound to a metal surface/electrode with work function $\phi$. In forming the metalmolecule bond, the HOMO resonance of the molecule shifts and hybridizes.

the $\pi$-electrons was derived, keeping all interaction terms up to the quadrupole-quadrupole interaction. The minimal set of parameters necessary to characterize the $\pi$-electron system consists of the $\pi$-orbital on-site repulsion $U_{0}$, the $\pi$-orbital quadrupole moment $Q$, the nearest-neighbor $\pi$ $\pi$ hybridization $t$, and the dielectric constant $\epsilon$, which accounts for the polarizability of the $\sigma$-electrons, which are not included explicitly in the calculation. The values $U_{0}=8.9 \mathrm{eV}, Q=0.67 e \AA^{2}, t=2.64 \mathrm{eV}$, and $\epsilon=1.63$ were determined by fitting to the gas-phase spectrum of benzene.[14] In the molecular junction, screening of intramolecular Coulomb interactions by the nearby metal electrodes [15, 16] was included via the image charge method, with no additional adjustable parameters.

\section{JUNCTION ENSEMBLE}

In this article, we consider junctions in which two macroscopic multi-channel leads each couple to several atomic orbitals of a single molecule. In order to calculate the distribution of transmission eigenvalues, it is first necessary to construct a physical ensemble of junctions. Both the lead-molecule coupling and the electrode geometry [17] vary over the ensemble of junctions produced in an experiment [3]. The lead-molecule coupling involves both screening [15, 16] and hybridization of the molecular and metallic states, described by the matrix $\Sigma_{\mathrm{T}}$ (see Fig. 2). We assume both effects are dominated by the interaction of the molecule with a single Pt atom at the tip of each electrode, as illustrated in Fig. 1 Since $\Sigma_{\mathrm{T}}$ depends exponentially on the tip-molecule distance, we assume its variation is most important, and keep screening fixed over the ensemble of junctions. Moreover, we neglect the real part of $\Sigma_{\mathrm{T}}$ and focus on the variation of the tunneling-width matrices $\Gamma_{\alpha}$. The variation of electrode geometry leads to a variation of the work function $\phi$ and density of states (DOS) of the leads (the latter also contributes to the variation of $\Gamma_{\alpha}$.) 
We thus assume the ensemble of junctions can be modeled adequately through variations of $\Gamma_{\alpha}$ and $\phi$ only [18].

\section{Molecule-electrode Coupling}

In order to determine the tunneling-width matrices $\Gamma_{\alpha}$ for a SMJ, we first consider the details of a single benzene molecule adsorbed on a $\operatorname{Pt}(111)$ surface. This is the most stable Pt surface, and has been the subject of numerous investigations, where the observed binding energy for benzene ranges between $21 \mathrm{kcal} / \mathrm{mol}(0.91 \mathrm{eV} /$ molecule $)$ to $29 \mathrm{kcal} / \mathrm{mol}(1.26 \mathrm{eV} /$ molecule $)$ corresponding to the atop $(0)$ and bridge(30) bonding configurations, respectively [19-21]. As indicated schematically in Fig. 2] when a molecule binds with a metal surface, the relevant energy levels of the molecule shift and hybridize, forming bonding and anti-bonding states. These two effects contribute to the binding energy $\Delta E_{\mathrm{b}}=\Delta E_{\mathrm{vdW}}+\Delta E_{\mathrm{hyb}}$, where $\Delta E_{\mathrm{vdW}}=\left\langle H_{\mathrm{mol}}\right\rangle-\left\langle\tilde{H}_{\mathrm{mol}}\right\rangle$ is the van der Waals energy shift and $\Delta E_{\text {hyb }}$ is the hybridization energy. Here $H_{\text {mol }}$ is the gas-phase molecular Hamiltonian and $\tilde{H}_{\text {mol }}$ is the molecular Hamiltonian including screening from the $\mathrm{Pt}$ surface. Taking the benzene-Pt distance as $2.25 \AA$ and assuming the screening is dominated by the nearest $\mathrm{Pt}$ atom, we find that the HOMO-LUMO gap of benzene reduces from $10.05 \mathrm{eV}$ in the gas-phase to $7.52 \mathrm{eV}$ on $\mathrm{Pt}(111)$ and $\Delta E_{\mathrm{vdW}}=0.49 \mathrm{eV}$. This implies $\Delta E_{\mathrm{hyb}} \leq 0.77 \mathrm{eV}$.

Since the metallic work function $\phi$ lies between the HOMO and LUMO resonances, hybridization occurs via the virtual exchange of an electron or hole between the metal and the neutral molecule. Using second-order perturbation theory, we find:

$$
\begin{aligned}
\Delta E_{\mathrm{hyb}} & =\sum_{\nu \in \mathcal{H}_{N-1}} \int_{\mu}^{\infty} \frac{d E}{2 \pi} \frac{\operatorname{Tr}\left\{\Gamma(E) C\left(\nu, 0_{N}\right)\right\}}{E-E_{0_{N}}+E_{\nu}} \\
& +\sum_{\nu^{\prime} \in \mathcal{H}_{N+1}} \int_{-\infty}^{\mu} \frac{d E}{2 \pi} \frac{\operatorname{Tr}\left\{\Gamma(E) C\left(0_{N}, \nu^{\prime}\right)\right\}}{-E-E_{0_{N}}+E_{\nu^{\prime}}}
\end{aligned}
$$

where $\mu$ is the chemical potential of the lead metal, $\mathcal{H}_{N}$ is the $N$-particle molecular Hilbert space, and $0_{N}$ is the ground state of the $N$-particle manifold of the neutral molecule. At room temperature, the Pt DOS $g(E)$ is sharply peaked around the Fermi energy [22], allowing us to perform the energy integral in Eq. [5 using $\Gamma(E) \approx$ $\Gamma\left(\varepsilon_{F}\right) Z \delta\left(E-\varepsilon_{F}\right) / g\left(\varepsilon_{F}\right)$, where we take $Z=+4$ for a $\mathrm{Pt}$ atom and $g\left(\varepsilon_{F}\right)=2.88 / \mathrm{eV}$ [23]. The hybridization energy is thus determined by the tunneling-width matrix evaluated at the Pt Fermi level, $\Gamma\left(\varepsilon_{F}\right) \equiv \Gamma$. Using $\phi_{\operatorname{Pt}(111)}=5.93 \mathrm{eV}$ [24] and the chemical potential of benzene $\mu_{0}=(\mathrm{IE}+\mathrm{EA}) / 2=-$ $4.06 \mathrm{eV}[25,26]$ (which is unaffected by screening), we find $\operatorname{Tr}\{\Gamma\} \leq 21.6 \mathrm{eV}$ to fit the hybridization energy for benzene adsorbed on Pt.
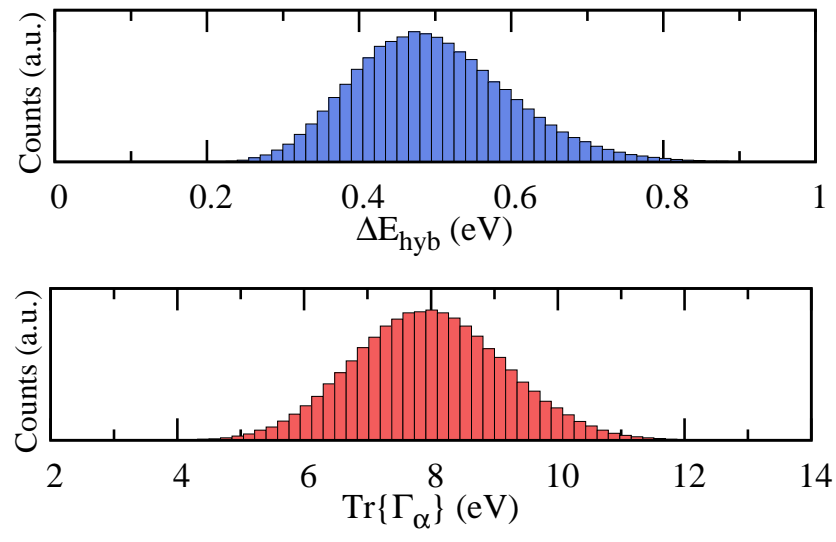

FIG. 3. Distributions of hybridization energy (top) and tunnelingwidth matrix trace (bottom) for a Pt-benzene-Pt junction. The $99^{\text {th }}$-percentile values for $\Delta E_{\text {hyb }}$ and $\operatorname{Tr}\left\{\Gamma_{\alpha}\right\}$ are $0.77 \mathrm{eV}$ and $10.82 \mathrm{eV}$, respectively. Here $\alpha=1,2$ labels the lead-molecule contacts.

\section{RESULTS AND DISCUSSION}

\section{Pt-benzene-Pt Junctions}

Let us now consider lead-molecule hybridization in a Pt-benzene-Pt junction. Screening from two metal electrodes further reduces the HOMO-LUMO gap to $6.46 \mathrm{eV}$. Since the most favorable binding of benzene on the closestpacked $\mathrm{Pt}(111)$ surface gives $\Delta E_{\mathrm{hyb}}=0.77 \mathrm{eV}$, we assume this is essentially an upper bound on hybridization in a SMJ, where the bonding is more random. We wish to study the dependence of the transmission eigenvalue distribution on the number of bonds formed with each electrode. For $M$ covalent bonds between a macroscopic lead and a molecule with $P$ atomic orbitals, $\Gamma$ is a rank- $M$ matrix, which can be represented as

$$
\Gamma=\sum_{m=1}^{M} \gamma_{m}^{\dagger} \gamma_{m},
$$

where $\gamma_{m}$ are linearly-independent real row vectors of dimension $P$, representing linear combinations of the atomic orbitals of the molecule [27]. Our approach is to populate the elements of $\gamma_{m}$ from a uniform random distribution on the interval $[-A, A]$. The bonding ensemble corresponds to a random walk of $M$ steps in a $P$-dimensional space. The distributions of $\Delta E_{\text {hyb }}$ and $\operatorname{Tr}\left\{\Gamma_{\alpha}\right\}$ shown in Fig. 3 have long Gaussian tails, so the value $A=0.82 \mathrm{eV}$ was chosen to fix the $99^{\text {th }}$-percentile of $\Delta E_{\text {hyb }}$ at $0.77 \mathrm{eV}$ [28]. The $99^{\text {th }}$-percentile of $\operatorname{Tr}\left\{\Gamma_{\alpha}\right\}$ is $10.82 \mathrm{eV}$ which, per orbital, is nearly $3 \times$ the coupling found for a Au-BDT-Au junction [8].

In addition to sampling a variety of bonding configurations, we assume the ensemble of junctions samples all possible Pt surfaces. The work function of $\mathrm{Pt}$ ranges from $5.93 \mathrm{eV}$ to $5.12 \mathrm{eV}$ for the (111) and (331) surfaces, respec- 


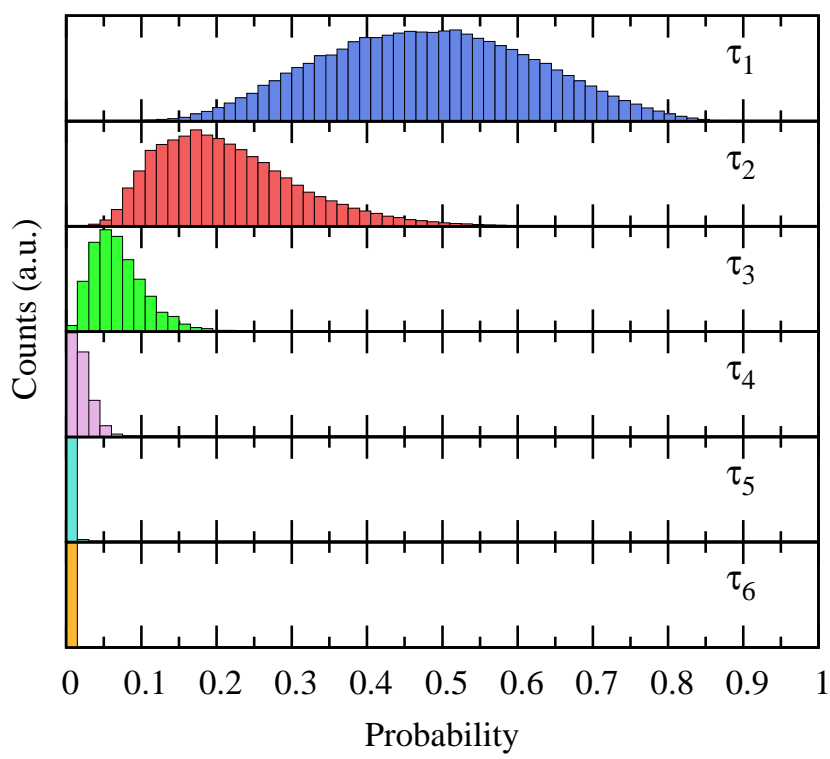

FIG. 4. Calculated transmission eigenvalue distributions for an ensemble of $24 \times 10^{4} \mathrm{Pt}$-benzene-Pt junctions. 3,000 bonding configurations $\times 80 \mathrm{Pt}$ surfaces were included. Each lead was assumed to form $M=6$ bonds with the molecule. There are two dominant transmission channels arising from the two-fold degenerate HOMO resonance closest to the Pt Fermi level, with a small but experimentally resolvable third channel arising from further off-resonant tunneling.

tively [24], so that

$$
-1.88 \mathrm{eV} \leq \mu_{\mathrm{Pt}}-\mu_{0} \leq-1.07 \mathrm{eV},
$$

and we assume a uniform distribution of $\mu_{\mathrm{Pt}}$ on this interval.

The transmission eigenvalue distributions are shown in Fig. 4 for an ensemble of $24 \times 10^{4} \mathrm{Pt}-$ benzene-Pt junctions. Each lead was assumed to form $M=6$ bonds with the molecule. Despite the existence of six covalent bonds between the molecule and each lead, there are only two dominant transmission channels, which arise from the twofold degenerate HOMO resonance closest to the Pt Fermi level. There is also a small but experimentally resolvable third channel arising from further off-resonant tunneling. This channel is non-negligible because of the very large lead-molecule coupling $\Gamma$ in the Pt-benzene junction. Explicit calculations with smaller values of $\operatorname{Tr}\{\Gamma\}$ yielded only two non-negligible transmission eigenvalues. Thus, for metals with a smaller DOS at the Fermi level, such as $\mathrm{Cu}, \mathrm{Ag}$, or $\mathrm{Au}$, junctions with benzene would be expected to exhibit only two measurable transmission channels.

The calculated transmission eigenvalue distribution shown in Fig. 4]is consistent with the experiment [3], which determined the transmission eigenvalues for three particular junctions: $\left\{\tau_{n}\right\}=\{0.68,0.40\},\{0.36,0.25,0.10\}$, $\{0.20\}$, where a third channel was observable only once. The conductance histogram for the same ensemble of junctions is shown in Fig. 5 The peak conductance value is

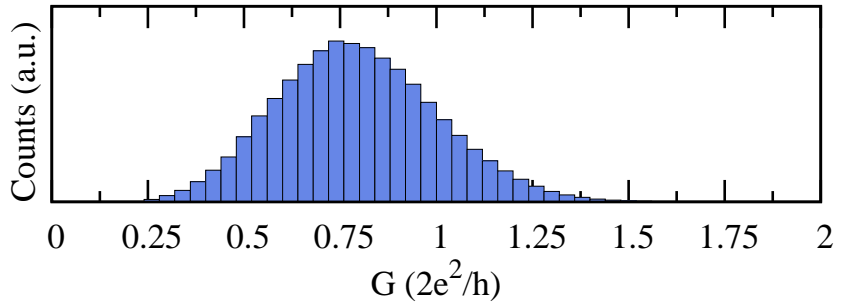

FIG. 5. Calculated conductance histogram for the same ensemble of Pt-benzene-Pt junctions discussed in Fig. 4, where $G=\left(2 e^{2} / h\right) \sum_{n} \tau_{n}$.

some $20 \%$ less than that reported experimentally [3]. This discrepancy might be attributable, in part, to the inclusion of a small fraction of Pt-Pt junctions in the experimental histogram.

In addition to the ensemble of junctions shown in Fig. 4. we also investigated ensembles of junctions with $M_{\alpha}=$ $1, \ldots, 6$, including the case $M_{1} \neq M_{2}$. Consistent with the discussion in Refs. [3, 5], we find that the total number of nonzero transmission eigenvalues is $M_{\min }=$ $\min \left\{M_{1}, M_{2}\right\}$. However, whenever $M_{\text {min }} \geq 2$ there are always two dominant transmission channels, and the total transmission probability does not increase appreciably beyond $M_{\min }=2$.

The above analysis demonstrates that the two dominant transmission channels evolve from the two-fold degenerate HOMO resonance in Eq. 3 as the lead-molecule coupling $\Sigma_{\mathrm{T}}$ is turned on. For finite $\Sigma_{\mathrm{T}}$, the poles of $G_{\mathrm{mol}}$ are mixed by Dyson's equation (Eq. 2), making it problematic to decompose the transmission eigenvalues into separate contributions from each molecular resonance [5]. Alternatively, the projections of the transmission eigenvectors onto the molecular resonances can be computed [4]. Because an "extended molecule" is often used in densityfunctional calculations to account for charge transfer between the molecule and electrodes, it is difficult if not impossible to interpret these contributions in terms of the resonances of the molecule itself [4]. Since charging effects in SMJs are well-described in our many-body theory [8], there is no need to utilize an "extended molecule," so the projections of the transmission eigenvectors onto the molecular resonances can be determined unambiguously (see Methods). We find that the mean-square projections of the first and second transmission channels in Fig. 4 4 onto the benzene HOMO resonance are $87 \%$ and $71 \%$, respectively, confirming the conclusion that these eigenchannels correspond to tunneling primarily through the HOMO resonance.

\section{Pt-butadiene-Pt Junctions}

To test our hypothesis that the number of dominant transmission channels is limited by the degeneracy of the 


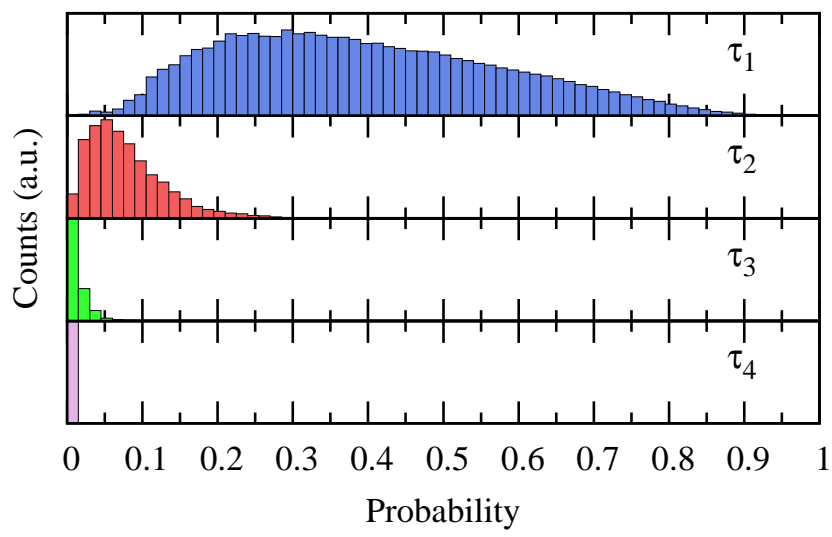

FIG. 6. Transmission eigenvalue distributions for an ensemble of $24 \times 10^{4} \mathrm{Pt}$-butadiene-Pt junctions. 3,000 bonding configurations $\times 80 \mathrm{Pt}$ surfaces were included. Each lead was assumed to form $M=4$ bonds with the molecule. The linear butadiene $\left(\mathrm{C}_{4} \mathrm{H}_{6}\right)$ molecule lacks the orbital degeneracy of the benzene ring and consequently exhibits only 1 dominant channel. A second channel due to further off-resonant tunneling may be experimentally resolvable. The total conductance distribution peaks around $\sim 0.4 \mathrm{G}_{0}$.

most relevant molecular orbital, we have investigated transmission eigenvalue distributions for Pt-butadiene-Pt junctions. Butadiene is a linear conjugated polymer, lacking the discrete (six-fold) rotational symmetry of benzene. Since the molecular orbitals of butadiene are nondegenerate, we anticipate a single dominant transmission channel. Using the same per-orbital hybridization as for benzene gives $\operatorname{Tr}\left\{\Gamma_{\alpha}\right\} \leq 7.21 \mathrm{eV}$. The image charge method gives $\Delta E_{\mathrm{vdW}}=0.52 \mathrm{eV}$ and we find $\Delta E_{\mathrm{hyb}} \leq 0.59 \mathrm{eV}$, so that $\Delta E_{\mathrm{b}} \leq 1.12 \mathrm{eV} \equiv 107.7 \mathrm{~kJ} / \mathrm{mol}$, in line with existing values reported in the literature [30]. The range of $\mathrm{Pt}$ work functions for all possible Pt surfaces gives a chemical potential range of

$$
-1.70 \mathrm{eV} \leq \mu_{\mathrm{Pt}}-\mu_{0} \leq-0.89 \mathrm{eV} .
$$

Despite forming four bonds with each electrode, it is evident from Fig. 6 that the Pt-butadiene-Pt junction has a single dominant transmission channel. The mean-square projection of this transmission channel onto the nondegenerate butadiene HOMO resonance is $80 \%$ (see Methods).

\section{CONCLUSIONS}

In conclusion, we find that the number of dominant transmission channels in a SMJ is equal to the degeneracy of the molecular orbital closest to the metal Fermi level. Transmission eigenvalue distributions were calculated for Pt-benzene-Pt and Pt-butadiene-Pt junctions using realistic state-of-the-art many-body techniques. In both cases, transmission occurs primarily through the HOMO resonance, which lies closest to the Pt Fermi level, resulting in two dominant transmission channels for benzene (2-fold degenerate HOMO) and a single dominant transmission channel for butadiene (non-degenerate HOMO). Our results for the transmission channel distributions of Ptbenzene-Pt junctions (see Fig. (4) are in quantitative agreement with experiment [3].

Despite the larger number of states available for tunneling transport in SMJs, we predict that the number of transmission channels is typically more limited than in single-atom contacts because molecules are less symmetrical than atoms. Nonetheless, certain highly-symmetric molecules exist that should permit several dominant transmission channels. For example, the $\mathrm{C}_{60}$ molecule possesses icosahedral symmetry, and has a 5-fold degenerate HOMO resonance and 3-fold degenerate LUMO resonance [31]. $\mathrm{C}_{60}$-based SMJs with gold electrodes have been fabricated, and shown to exhibit fascinating electromechanical [32] and spin-dependent [33] transport properties. For $\mathrm{Pt}-\mathrm{C}_{60}-\mathrm{Pt}$ junctions, we predict five dominant transmission channels stemming from the $\mathrm{C}_{60} \mathrm{HOMO}$ resonance, which lies closest to the Pt Fermi level [34, 35].

\section{METHODS}

\section{Decomposing transmission channels in terms of molecular resonances}

In order to understand how transport in a SMJ is determined by the chemical properties of the molecule, it would be desirable to express the transmission eigenchannels in terms of molecular resonances [4, 5]. The transmission eigenvalues $\tau_{n}$ and eigenvectors $|n\rangle$ are solutions of the equation

$$
T|n\rangle=\tau_{n}|n\rangle,
$$

where $T$ is the transmission matrix given by Eq. 1 By definition, $|n\rangle$ is a linear combination of the atomic orbitals of the molecule. In an effective single-particle model [4], $|n\rangle$ may also be expressed as a linear combination of molecular orbitals $\left|\phi_{j}\right\rangle$

$$
|n\rangle=\sum_{j} \alpha_{n}^{j}\left|\phi_{j}\right\rangle
$$

Here $\left|\alpha_{n}^{j}\right|^{2}$ can be identified as the contribution of the $j$ th molecular orbital to the $n$th transmission channel [4], which can be conveniently expressed in terms of the projection operator $\hat{P}_{j}=\left|\phi_{j}\right\rangle\left\langle\phi_{j}\right|$ as $\left|\alpha_{n}^{j}\right|^{2}=\left\langle n\left|\hat{P}_{j}\right| n\right\rangle$.

In the many-body problem, there is no orthonormal set of "molecular orbitals"; rather each molecular resonance of energy $E_{\nu^{\prime}}-E_{\nu}$ corresponds to a transition $\nu \rightarrow \nu^{\prime}$ between an $N$-body and an $(N+1)$-body molecular eigenstate [see Eqs. 3] and 4]. The projection operator onto a molecular resonance is

$$
\hat{P}_{\nu \rightarrow \nu^{\prime}} \equiv \frac{C\left(\nu, \nu^{\prime}\right)}{\operatorname{Tr}\left\{C\left(\nu, \nu^{\prime}\right)\right\}},
$$


where $C\left(\nu, \nu^{\prime}\right)$ is given by Eq. 4. The absolute square projection of the $n$th transmission eigenvector onto the resonance $\nu \rightarrow \nu^{\prime}$ is given by

$$
\left|\alpha_{n}^{\nu \rightarrow \nu^{\prime}}\right|^{2}=\left\langle n\left|\hat{P}_{\nu \rightarrow \nu^{\prime}}\right| n\right\rangle .
$$

A necessary condition to identify an eigenchannel $|n\rangle$ with transmission through a particular molecular resonance is $\left|\alpha_{n}^{\nu \rightarrow \nu^{\prime}}\right|^{2} \approx 1$.

The above procedure is in principle straightforward to implement in an effective single-particle model based on density functional theory (DFT)[4]. However, in practice, an "extended molecule" must be used in DFT calculations to account for charge transfer between the molecule and electrodes. This is because current implementations of DFT fail to account for the particle aspect of the electron [10, 36-38], i.e., the strong tendency for the electric charge on the molecule within the junction to be quantized in integer multiples of the electron charge $e$. Analyzing transport in terms of extended molecular orbitals has unfortunately proven problematic. For example, the resonances of the extended molecule in Ref. 4 apparently accounted for less than $9 \%$ of the current through the junction.

Since charging effects in SMJs are well-described in our many-body theory [8], there is no need to utilize an "extended molecule," so the projections of the transmission eigenvectors onto the molecular resonances can be determined directly from Eq. 12.

\section{Benzene resonances}

The neutral ground state of benzene is nondegenerate, while the HOMO and LUMO resonances are both doubly degenerate due to the (six-fold) rotational symmetry of the molecule. To be consistent with the discussion of Ref. 3, the additional two-fold spin degeneracy of each resonance is considered implicit.

We define the following projection operators:

$$
\begin{aligned}
\hat{P}_{\mathrm{HOMO}} & \equiv \sum_{\nu \in 0_{5}} \hat{P}_{\nu \rightarrow 0_{6}}, \\
\hat{P}_{\mathrm{LUMO}} & \equiv \sum_{\nu^{\prime} \in 0_{7}} \hat{P}_{0_{6} \rightarrow \nu^{\prime}}, \\
\hat{P}_{\perp} & \equiv \mathbf{1}-\hat{P}_{\mathrm{HOMO}}-\hat{P}_{\mathrm{LUMO}},
\end{aligned}
$$

where $0_{N}$ is the ground state with $N \pi$-electrons and 1 is the six-dimensional unit matrix in the space of $\pi$ orbitals. $\hat{P}_{\mathrm{HOMO}}$ and $\hat{P}_{\mathrm{LUMO}}$ are projection operators onto the two-dimensional subspaces spanned by the HOMO and LUMO resonances, respectively. Eqs. 13, 15 define a complete, orthogonal set of projection operators in sixdimensions with the properties

$$
\begin{aligned}
\sum_{j} \hat{P}_{j} & =\mathbf{1}, \\
\hat{P}_{i} \hat{P}_{j} & =\hat{P}_{i} \delta_{i j} .
\end{aligned}
$$

In particular, Eq. 17 implies that the HOMO and LUMO subspaces of benzene are orthogonal. The absolute square of the projection of the $\mathrm{n}^{\text {th }}$ transmission eigenvector onto the subspace spanned by $\hat{P}_{j}$ is

$$
\left|\alpha_{n}^{j}\right|^{2}=\left\langle n\left|\hat{P}_{j}\right| n\right\rangle
$$

These coefficients satisfy the condition

$$
\sum_{j}\left|\alpha_{n}^{j}\right|^{2}=1
$$

where the sum runs over $j=\mathrm{HOMO}, \mathrm{LUMO}, \perp$.

Fig. 7 shows the mean-square projections $\left\langle\left|\alpha_{n}^{j}\right|^{2}\right\rangle$ of the transmission eigenvectors onto (a) the benzene HOMO resonance; (b) the benzene LUMO resonance; and (c) the two-dimensional subspace orthogonal to both the HOMO and LUMO resonances, as a function of electrode chemical potential for the same ensemble of Pt-benzene-Pt junctions discussed in Fig. 4 We find that the mean-square projections of the first and second transmission channels onto the benzene HOMO resonance are $87 \%$ and $71 \%$, respectively, confirming the conclusion that these eigenchannels correspond to tunneling primarily through the HOMO resonance.

Midway between the HOMO and LUMO resonances at $\mu=\mu_{0}$, the first two transmission channels have mean-square projections of $\approx 0.5$ onto both the HOMO and LUMO resonances, consistent with the expectation that the HOMO and LUMO resonances should contribute equally to transmission. The remaining channels do not have negligible overlap with the HOMO resonance, but instead cluster around $\left\langle\left|\alpha_{n}^{\mathrm{HOMO} O}\right|^{2}\right\rangle \sim 0.3$. The transmission channels with $\left\langle\tau_{n}\right\rangle \ll 1$ correspond to contributions from several far off-resonant poles, each of which has some overlap with the HOMO resonance due to the overcompleteness of the projectors $\hat{P}_{\nu \rightarrow \nu^{\prime}}$ [39]. They are essentially random unit vectors in the six-dimensional space of benzene $\pi$-orbitals, whose mean-square overlap with the twodimensional HOMO subspace should be $\left\langle\left|\alpha_{n}^{\mathrm{HOMO}}\right|^{2}\right\rangle=$ $2 / 6=1 / 3$.

The first two transmission channels only have an appreciable overlap with the subspace orthogonal to the HOMO and LUMO resonances in the vicinity of a pronounced dip in the transmission spectrum at $\mu-\mu_{0} \approx \pm 3.5 \mathrm{eV}$. 

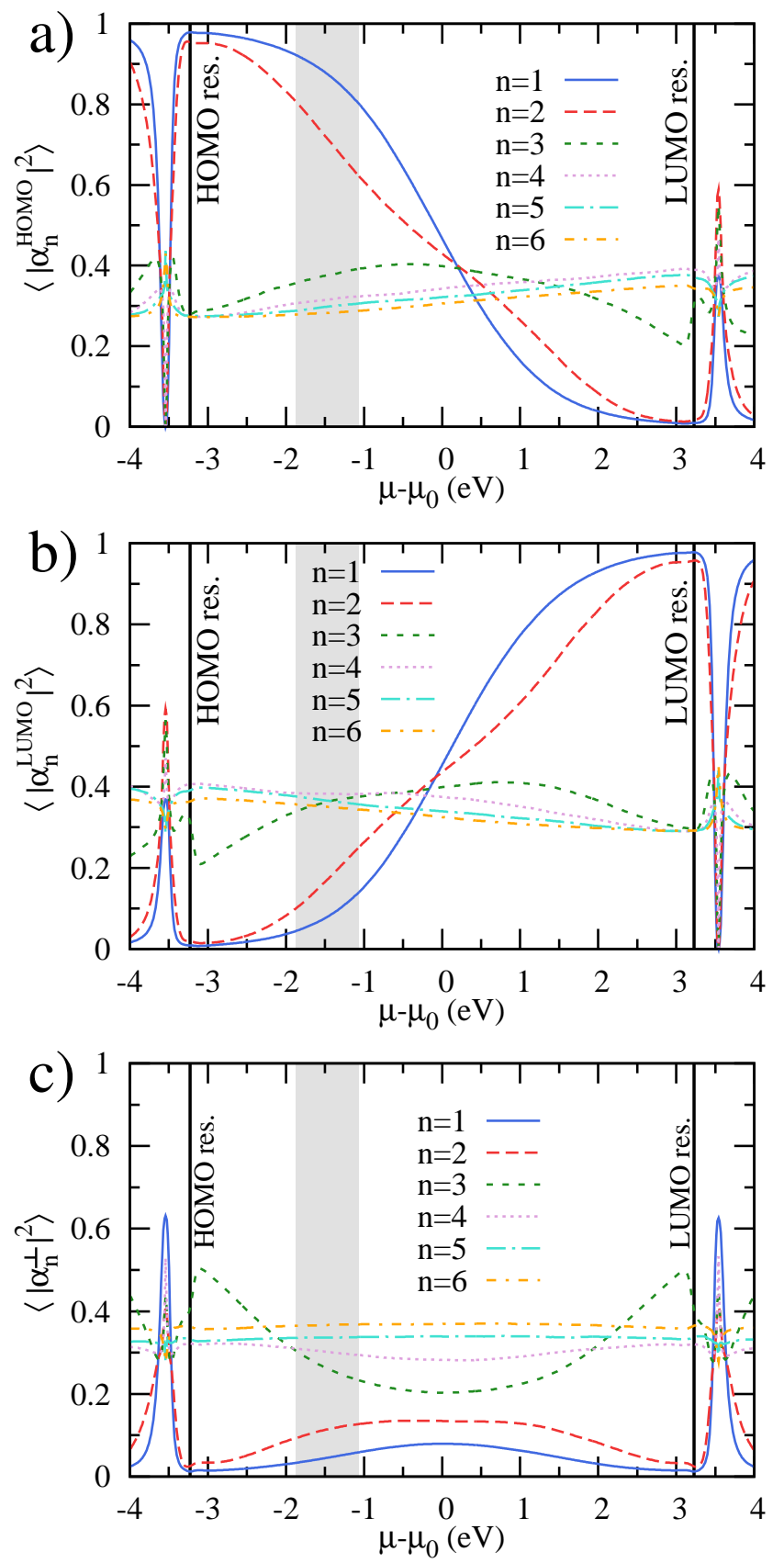

FIG. 7. The mean-square projections $\left|\alpha_{n}^{j}\right|^{2}=\left\langle n\left|\hat{P}_{j}\right| n\right\rangle$ of the transmission eigenvectors onto the two-dimensional subspaces (a) spanned by the benzene HOMO resonance; (b) spanned by the benzene LUMO resonance; and (c) orthogonal to the HOMO and LUMO subspaces, for the same ensemble of Pt-benzene-Pt junctions discussed in Fig. 4. For the range of possible chemical potentials of Pt electrodes, $-1.88 \mathrm{eV} \leq \mu_{\mathrm{Pt}}-\mu_{0} \leq-1.07 \mathrm{eV}$ indicated by the grey boxes in each subfigure, the first two channels have very strong overlap with the HOMO resonance: $87 \%$ and $71 \%$, respectively.

\section{Butadiene resonances}

The neutral ground state of butadiene is nondegenerate, and the HOMO and LUMO resonances have no orbital degeneracy. The projection operators onto the HOMO and LUMO resonances of butadiene are

$$
\begin{aligned}
\hat{P}_{\mathrm{HOMO}} & \equiv \hat{P}_{0_{3} \rightarrow 0_{4}}, \\
\hat{P}_{\mathrm{LUMO}} & \equiv \hat{P}_{0_{4} \rightarrow 0_{5}},
\end{aligned}
$$

respectively. Fig. 8 shows the mean-square projections of the transmission eigenvectors onto the nondegenerate butadiene HOMO and LUMO resonances as a function of electrode chemical potential for the same ensemble of Ptbutadiene-Pt junctions discussed in Fig. 6 In this case, the single dominant channel has a strong overlap with the nondegenerate $\mathrm{HOMO}$ resonance: $\left\langle\left|\alpha_{1}^{\mathrm{HOMO}}\right|^{2}\right\rangle=0.80$ when averaged over the range $-1.70 \mathrm{eV} \leq \mu_{\mathrm{Pt}}-\mu_{0} \leq-0.89 \mathrm{eV}$, while the far off-resonant channels with $\left\langle\tau_{n}\right\rangle \ll 1$ have $\left\langle\left|\alpha_{n}^{\mathrm{HOMO}}\right|^{2}\right\rangle \sim 1 / 4$, as expected based on the arguments given above.

* justinb@email.arizona.edu

[1] Cron, R.; Goffman, M. F.; Esteve, D.; Urbina, C. MultipleCharge-Quanta Shot Noise in Superconducting Atomic Contacts. Phys. Rev. Lett. 2001, 86, 4104-4107.

[2] Scheer, E.; Agraït, N.; Cuevas, J. C.; Levy Yeyati, A.; Ludoph, B.; Martín-Rodero, A.; Rubio Bollinger, G.; van Ruitenbeek, J. M.; Urbina, C. The signature of chemical valence in the electrical conduction through a single-atom contact. Nature 1998, 394, 154-157.

[3] Kiguchi, M.; Tal, O.; Wohlthat, S.; Pauly, F.; Krieger, M.; Djukic, D.; Cuevas, J. C.; van Ruitenbeek, J. M. Highly conductive molecular junctions based on direct binding of benzene to platinum electrodes. Phys. Rev. Lett. 2008, 101.

[4] Heurich, J.; Cuevas, J. C.; Wenzel, W.; Schön, G. Electrical Transport through Single-Molecule Junctions: From Molecular Orbitals to Conduction Channels. Phys. Rev. Lett. 2002, 88, 256803.

[5] Solomon, G. C.; Gagliardi, A.; Pecchia, A.; Frauenheim, T.; Di Carlo, A.; Reimers, J. R.; Hush, N. S. Molecular Origins of Conduction Channels Observed in Shot-Noise Measurements. Nano Lett. 2006, 6, 2431-2437.

[6] Often, the transmission matrix is expressed in the basis of propagating states in the leads $\tilde{T}=t t^{\dagger}$, where $t=i \gamma_{1} G \gamma_{2}^{\dagger}$ and $\Gamma_{\alpha}=\gamma_{\alpha}^{\dagger} \gamma_{\alpha}$, rather than in the molecular basis. It is straightforward to show that $T$ and $\tilde{T}$ have the same nonzero eigenvalues: Suppose $\tilde{T}|\tilde{n}\rangle=\tau_{n}|\tilde{n}\rangle$. Let $|n\rangle=\gamma_{1}^{\dagger}|\tilde{n}\rangle$. Then $T|n\rangle=\gamma_{1}^{\dagger} \tilde{T}|\tilde{n}\rangle=\tau_{n}|n\rangle$.

[7] Paulsson, M.; Brandbyge, M. Transmission eigenchannels from nonequilibrium Green's functions. Phys. Rev. B 2007, 76, 115117.

[8] Bergfield, J. P.; Stafford, C. A. Many-body theory of electronic transport in single-molecule heterojunctions. Phys. Rev. B 2009, 79, 245125. 

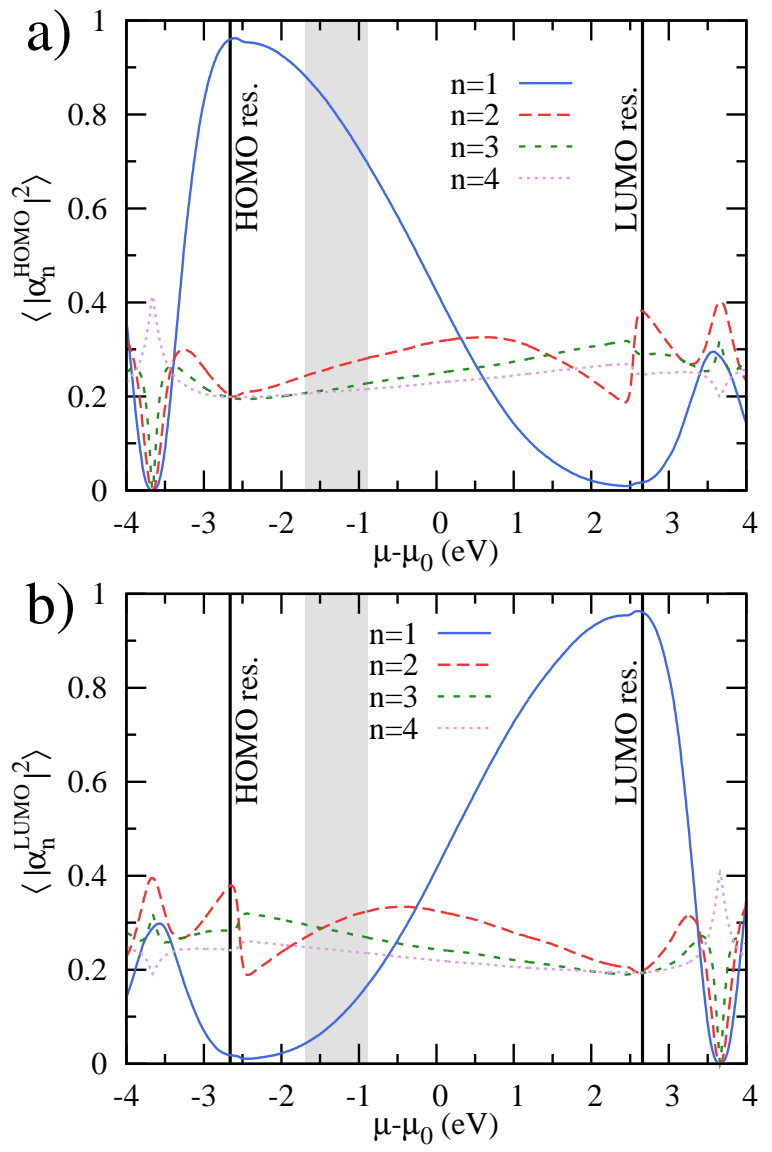

[15] Neaton, J. B.; Hybertsen, M. S.; Louie, S. G. Renormalization of Molecular Electronic Levels at Metal-Molecule Interfaces. Phys. Rev. Lett. 2006, 97, 216405.

[16] Thygesen, K. S.; Rubio, A. Renormalization of Molecular Quasiparticle Levels at Metal-Molecule Interfaces: Trends across Binding Regimes. Phys. Rev. Lett. 2009, 102, 046802.

[17] Bürki, J.; Goldstein, R. E.; Stafford, C. A. Quantum Necking in Stressed Metallic Nanowires. Phys. Rev. Lett. 2003, 91, 254501.

[18] The variation of $\phi$ has an effect similar to any variation of $\operatorname{Re} \Sigma_{\mathrm{T}}$

[19] Cruz, M. T. d. M.; Carneiro, J. W. d. M.; Aranda, D. A. G.; Bãhl, M. Density Functional Theory Study of Benzene Adsorption on Small Pd and Pt Clusters. J. Phys. Chem. C 2007, 111, 11068-11076.

[20] Morin, C.; Simon, D.; Sautet, P. Density-Functional Study of the Adsorption and Vibration Spectra of Benzene Molecules on Pt(111). J. Phys. Chem. B 2003, 107, 29953002.

[21] Saeys, M.; Reyniers, M.-F.; Marin, G. B.; Neurock, M. Density Functional Study of Benzene Adsorption on $\operatorname{Pt}(111) . J$. Phys. Chem. B 2002, 106, 7489-7498.

[22] Kleber, R. On the electronic density of states of the transition metals. Z. Phys. A: Hadrons Nucl. 1973, 264, 301-308.

[23] Kittel, C. New York: Wiley, 1976, 5th ed.; John Wiley and Sons, Inc., 1976.

[24] Lide et al., D. R., Ed. CRC Handbook of Chemistry and Physics; CRC Press: Boca Raton, Fla., 2005.

[25] Mikaya, A. I.; Trusova, E. A.; Zaikin, V. G.; Karaulova, E. N.; Petrova, L. M. Ionization energy and appearance energy in organic chemistry. Russ. Chem. B+ 1982, 31, 1319-1320.

FIG. 8. The mean-square projections $\left|\alpha_{n}^{j}\right|^{2}=\left\langle n\left|\hat{P}_{j}\right| n\right\rangle$ of the transmission eigenvectors onto (a) the butadiene HOMO resonance; and (b) the butadiene LUMO resonance, for the same ensemble of Pt-butadiene-Pt junctions discussed in Fig. 6 The dominant channel has a strong overlap ( $80 \%$ mean-square) with the HOMO resonance for the range of possible chemical potentials of Pt electrodes, $-1.70 \mathrm{eV} \leq \mu_{\mathrm{Pt}}-\mu_{0} \leq-0.89 \mathrm{eV}$, indicated on each subfigure by a solid grey box.

[9] Djukic, D.; van Ruitenbeek, J. M. Shot Noise Measurements on a Single Molecule. Nano Lett. 2006, 6, 789-793.

[10] Geskin, V.; Stadler, R.; Cornil, J. Multideterminant assessment of mean-field methods for the description of electron transfer in the weak-coupling regime. Phys. Rev. B 2009, 80, 085411.

[11] Bergfield, J. P.; Stafford, C. A. Thermoelectric Signatures of Coherent Transport in Single-Molecule Heterojunctions. Nano Lett. 2009, 9, 3072-3076.

[12] Bergfield, J. P.; Solis, M. A.; Stafford, C. A. Giant Thermoelectric Effect from Transmission Supernodes. ACS Nano 2010, 4, 5314-5320.

[13] Bergfield, J. P.; Jacquod, P.; Stafford, C. A. Coherent destruction of Coulomb blockade peaks in molecular junctions. Phys. Rev. B 2010, 82, 205405.

[14] Castleton, C. W. M.; Barford, W. Screening and the quantitative pi-model description of the optical spectra and polarizations of phenyl based oligomers. J. Chem. Phys. 2002, 117, 3570-3582.

[26] Janousek, B. K.; Brauman, J. I. In Gas Phase Ion Chemistry; Bowers, M., Ed.; Academic Press: New York, 1979.

[27] $\Gamma$ is a real symmetric matrix for systems with time-reversal invariance.

[28] Very similar results for transport are obtained if the $95^{\text {th }}$ percentile is used. Hybridization can be enhanced in the SMJ due to mesoscopic fluctuations [29] in the lead DOS.

[29] Stafford, C. A.; Kassubek, F.; Bürki, J.; Grabert, H. Universality in Metallic Nanocohesion: A Quantum Chaos Approach. Phys. Rev. Lett. 1999, 83, 4836-4839.

[30] Valcarcel, A.; Clotet, A.; Ricart, J. M.; Delbecq, F.; Sautet, P. Comparative DFT study of the adsorption of 1,3butadiene, 1-butene and 2-cis/trans-butenes on the $\operatorname{Pt}(111)$ and $\operatorname{Pd}(111)$ surfaces. Surf. Sci. 2004, 549, 121-133.

[31] Iwasa, Y.; Takenobu, T. Superconductivity, MottHubbard states, and molecular orbital order in intercalated fullerides. J. Phys.: Condens. Matter 2003, 15, R495.

[32] Park, H.; Park, J.; Lim, A. K. L.; Anderson, E. H.; Alivisatos, A. P.; McEuen, P. L. Nanomechanical oscillations in a single- $\mathrm{C}_{60}$ transistor. Nature 2000, 407, 57-60.

[33] Yu, L.; Natelson, D. The Kondo effect in C-60 singlemolecule transistors. Nano Lett. 2004, 4, 79-83.

[34] Lichtenberger, D. L.; Nebesny, K. W.; Ray, C. D.; Huffman, D. R.; Lamb, L. D. Valence and core photoelectron spectroscopy of C60, buckminsterfullerene. Chem. Phys. Lett. 1991, 176, 203 - 208.

[35] Kroto, H. W.; Allaf, A. W.; Balm, S. P. C60: Buckminsterfullerene. Chem. Rev. 1991, 91, 1213-1235. 
[36] Toher, C.; Filippetti, A.; Sanvito, S.; Burke, K. SelfInteraction Errors in Density-Functional Calculations of Electronic Transport. Phys. Rev. Lett. 2005, 95, 146402.

[37] Koentopp, M.; Burke, K.; Evers, F. Zero-bias molecular electronics: Exchange-correlation corrections to Landauer's formula. Phys. Rev. B 2006, 73, 121403.
[38] Muralidharan, B.; Ghosh, A. W.; Datta, S. Probing electronic excitations in molecular conduction. Phys. Rev. B 2006, 73, 155410.

[39] Note that there are 600 poles of the molecular Green's function (3) for a neutral benzene molecule, consistent with particle-number and $S_{z}$ selection rules (although some of these transitions are forbidden by the $S^{2}$ selection rule), vastly more than the six atomic orbitals in the basis set. 